\title{
An ontology-based methodology for supporting knowledge-intensive multi-discipline engineering processes
}

\author{
Thomas Moser Richard Mordinyi Stefan Biffl \\ Christian Doppler Laboratory \\ Software Engineering Integration for Flexible Automation Systems \\ Vienna University of Technology \\ \{thomas.moser, richard.mordinyi, stefan.biffl\}@tuwien.ac.at
}

\begin{abstract}
Software-intensive systems in business IT and industrial automation have become increasingly complex due to the need for more flexible system re-configuration, business and engineering processes. Systems and software engineering projects depend on the cooperation of experts from heterogeneous engineering disciplines using tools that were not designed to cooperate seamlessly. Current multi-discipline engineering is often ad hoc and fragile, making the evolution of tools and re-use of integration solutions across projects unnecessarily inefficient and risky.

This paper describes an ontology-based methodology, the socalled Engineering Knowledge Base (EKB), for engineering environment integration in multi-disciplinary engineering projects. The EKB stores explicit engineering knowledge to support access to and management of engineering models across tools and disciplines by providing a) data integration based on mappings between local and domain-level engineering concepts; b) transformations between local engineering concepts; and c) advanced applications built on these foundations, e.g., end-to-end analyses. As a result experts from different organizations may use their well-known tools and data models, and can access data from other tools in their syntax.

The methodology has been evaluated in an industrial application domain and initial evaluation results indicate an effort reduction for re-use in new engineering projects and finding defects earlier in the engineering process.
\end{abstract}

Keywords Knowledge Representation, Software Development Environments, Software and Systems Engineering, Multi-Discipline Engineering

\section{Introduction}

Software-intensive systems in business IT and industrial automation become increasingly complex due to the need for flexibility of business processes, system re-configuration, and engineering processes [14]. Such systems and software engineering projects bring together experts from several engineering domains and organizations, who work in a heterogeneous engineering environment with a wide range of models, processes, and tools that were originally

Permission to make digital or hard copies of all or part of this work for personal or classroom use is granted without fee provided that copies are not made or distributed for profit or commercial advantage and that copies bear this notice and the full citation on the first page. To copy otherwise, to republish, to post on servers or to redistribute to lists, requires prior specific permission and/or a fee.

2nd International Workshop on 'Ontology-Driven Software Engineering' October 17-21, Reno/Tahoe, Nevada, US.

Copyright (c) 2010 ACM [to be supplied]. . $\$ 10.00$ not designed to cooperate seamlessly. A core question is how to integrate data models across tools and domain boundaries. Current semantic engineering environment integration is often ad hoc and fragile, making the evolution of tools and re-use of integration solutions across projects risky [7][13].

In order to reach the common goal of developing software products in the engineering team, it is important to share the necessary knowledge for common work processes between engineering domain experts [14]. However, this knowledge is often only implicitly available and therefore inefficient to share, resulting in time-consuming repetitive tasks; often it is hard or even impossible to create and maintain common shared knowledge repositories. A method and platform for making expert knowledge explicit and efficiently shareable is needed in order to support quality and project managers in their data analyses based on engineering knowledge and concrete data in the engineering tool models, which is currently achieved using inefficient or fragile approaches.

An existing alternative solution is the usage of standards (e.g., $\mathrm{RUP}^{1}$, sysML ${ }^{2}$ ) for platforms, data models, modeling languages and tools in the development process [9][17]. This works well, if the standard is defined in an early phase of the project and if all project partners adhere to the standard, however, it is hard to define and maintain standards for cross-domain engineering, and even harder or nearly impossible for a larger number of project partners to agree on a standard, which usually takes longer than the time horizon of the project. Another alternative solution is the use of a common repository for collecting and storing the data of the single participants [3]. This is a typical solution for modern data-driven tool integration which well solves the challenges of persistence and versioning of data, but poses new challenges since the stored data often is hard to access and query [8][15].The ultimate alternative solution is the complete transformation between data models of tools [1], i.e., the translation of engineering model parts of one tool for work in another tool. While the advantage of this solution is the seamless cooperation between project partners using well-known and established tools and notations, the feasibility of this approach is hard to verify and the effort required for establishing the needed transformations is considerably high.

This paper proposes an ontology-based methodology, the socalled Engineering Knowledge Base (EKB) framework, for supporting engineering environment integration in multi-disciplinary engineering projects. Since standards are hard to apply in projects where experts from different organizations participate, who have invested into different kinds of local standards or approaches, these experts may use their well known local tools and data model, and

\footnotetext{
${ }^{1}$ Rational Unified Process: www.ibm.com/software/awdtools/rup

${ }^{2}$ Systems Modeling Language: www.sysml.org
} 
additionally can access data from other tools in their local syntax. The EKB is located on top of a common repository and stores explicit engineering knowledge to support access and management of engineering models across tools and disciplines by providing a) data integration by exploiting mappings between local and common engineering concepts; b) transformations between local engineering concepts by following these mappings; and c) advanced applications using these foundations, e.g., end-to-end analyses. Only a selection of the most relevant data elements to achieve interaction between engineering tools and experts is stored in the EKB in order to avoid time-consuming and effort-intensive transformations of full engineering models. As a result experts from different organizations may use their well known local tools and data model, and additionally can access data from other tools in their local syntax.

The research results have been evaluated in an industrial application domain, regarding effort, feasibility, performance, scalability, robustness and usability. Major results of this work are the feasibility of the EKB framework, i.e., the process, method and tool support is usable and useful across engineering domains, as well as better accuracy, effectiveness and efficiency. In addition, defects are found earlier in the engineering process, resulting in risks like errors or inconsistent entries in data models being mitigated earlier and more efficiently. Initial evaluation results indicate an effort reduction for re-use in new engineering projects and finding defects earlier in the engineering process. In addition, the engineers found the method usable and useful; furthermore, new kinds of analysis could be performed easily.

The remainder of this paper is structured as follows: Section 2 summarizes related work on semantic heterogeneity and on semantic integration. Section 3 describes the research challenges. Section 4 introduces the Engineering Knowledge Base (EKB) methodology and describes the generic framework architecture. Section 5 discusses the results of the EKB framework evaluation, and finally section 7 concludes and gives an outlook on future research.

\section{Related Work}

This section summarizes related work on semantic heterogeneity and on semantic integration. In the first subsection, problems, challenges and origins of semantic heterogeneity are explained, as well as solution approaches such as schema matching. In the second subsection, the research field of Semantic Integration is introduced, the different available approaches are classified and explained, and in addition application scenarios for the usage of ontologies for Semantic Integration are given.

\subsection{Semantic Heterogeneity}

Enterprises today are increasingly facing data management challenges that involve accessing and analyzing data residing in multiple sources, such as database systems, legacy systems, ERP systems and XML files and feeds. For example, in order for an enterprise to obtain a single view of customer data, they must tap into multiple databases. Similarly, to present a unified external view of their data, either to cooperate with a third party or to create an external facing web site, they must access multiple sources. There are many reasons for which data in enterprises resides in multiple sources. First, many data systems were developed independently for targeted business needs, but when the business needs changed, data needs to be shared between different parts of the organization. Second, enterprises acquire many data sources as a result of mergers and acquisitions [7].

The problem of reconciling schema heterogeneity has been a subject of research for decades, but solutions are few. The fundamental reason that makes semantic heterogeneity so hard is that the data sets were developed independently, and therefore varying structures were used to represent the same or overlapping concepts
[2][5]. In many cases, we are trying to integrate data systems that were developed for slightly (or vastly) different business needs. Hence, even if they model overlapping domains, they will model them in different ways. Differing structures are a byproduct of human nature people think differently from one another even when faced with the same modeling goal. From a practical perspective, one of the reasons that schema heterogeneity is difficult and time consuming is that it requires both domain and technical expertise: you need a person that understands the business meaning of each of the schemas being reconciled and people skilled in writing transformations (e.g., SQL or XQuery experts). While schema heterogeneity is challenging for humans, it is drastically more challenging for programs. A program is only given the two schemas to reconcile but those schemas are merely symbols. They do not capture the entire meaning or intent of the schemas; those are only in the minds of the designers [5].

\subsection{Semantic Integration}

Semantic Integration is defined as the solving of problems originating from the intent to share data across disparate and semantically heterogeneous data [7]. These problems include the matching of ontologies or schemas, the detection of duplicate entries, the reconciliation of inconsistencies, and the modeling of complex relations in different sources [13]. Over the last years, semantic integration became increasingly crucial to a variety of information-processing applications and has received much attention in the web, database, data-mining and AI communities. One of the most important and most actively studied problems in semantic integration is establishing semantic correspondences (also called mappings) between vocabularies of different data sources [5].

Goh [6] identified three main categories of semantic conflicts in the context of data integration that can appear: confounding conflicts, scaling conflicts, and naming conflicts. The use of ontologies as a solution option to semantic integration and interoperability problems has been studied over the last 10 years. Wache et al. [16] reviewed a set of ontology-based approaches and architectures that have been proposed in the context of data integration and interoperability.

Doan and Halevy [5] summarize the research on semantic integration in the database community. There, the matching of two database schemas requires deciding if any two elements of both schemas match, meaning that they refer to the same real-world concept. Typical challenges include the efficient extraction of semantic information, unreliable clues for matching schema elements (e.g., element names, types, data values, schema structures and integrity constraints), incomplete schema and data clues, and subjective matching depending on the application. Rule-based matching techniques use hand-crafted and/or probabilistic rules to exploit schema information for the identification of mappings. Rulebased matching techniques are relatively inexpensive and fairly fast since the typically operate only on schemas and not on data instances. But this is also their main drawback, as they cannot exploit data instances effectively, even though the instances can encode a wealth of information. Additionally, in many cases effective matching rules are simply too difficult to hand craft. Learningbased matching techniques consider a variety of machine learning techniques to exploit both schema and data information. There is also a growing realization that schema- and data-related evidence in two schemas being matched often is inadequate for the matching process, leading to the inclusion of external evidences beyond the two current schemas to the matching process. The key idea here is that a matching tool must be able to learn from past matches [7]. 


\section{Research Challenges}

The scope of this work is an engineering team consisting of experts from several engineering disciplines, who work on engineering process tasks with role-specific tools and systems that encapsulate engineering models and project data. As the engineers work together to deliver a product to the end user, they inevitably have to form common concepts on deliverables at interfaces between their work tasks. Such common concepts can be found in elements of requirements, design, and defect descriptions, which concern more than one role. Typical requirements for such engineering process tasks are low delay, i.e., in-time availability of information from other engineering tools and low effort for achieving the information exchange between the engineering tools.

Each engineering role (e.g., electrical engineer or software engineer) has a tailored tool set that works on data relevant to the engineer's tasks. In order to support the data exchange between these engineering tools, an additional component is needed. In a typical process step in the engineering process an engineer exports data from his tool to a transfer document (e.g., PDF of data table) and integrates this document in a common repository accessible by a set of partner engineering tools. The major challenges here are on the one hand side in the identification and description of tool data that should be extracted from tools and made available to other tools. On the other hand side, the data integration itself poses another huge challenge, since it is often not possible to agree on a common data schema agreed on by all tools, and additionally all engineers working with the tools want to stick with their well-known terms and notations. Finally, the re-use of at least parts of integration solutions for other projects with different project partners is mostly not possible. In order to support data exchange between these sets of partner engineering tools, transformations between the different notations and format of the particular partner engineering tools is needed. The major challenges of the transformation process are both the adaptation of transformation instructions to new or changed tool data structures which normally requires time-consuming manual human work, as well as the runtime performance of these transformation instructions. Using these foundations, i.e., export, integration and transformation, additional methods like Quality Assurance (QA) support or other advanced methods like model consistency checking or end-to-end analyses are allowed.

For these tasks, we propose to use the novel Engineering Knowledge Base (EKB) framework. In comparison to a simple data storage such as a common repository, a knowledge base stores information (i.e., the original data plus meta-data describing links between data elements or annotations of data elements using machine-understandable syntax which can be used to automate time-consuming tasks and support human experts in doing their work. The EKB stores explicit engineering knowledge to support access to and management of engineering models across tools and disciplines by providing (1) data integration by exploiting mappings between local and common engineering concepts; (2) transformations between local engineering concepts; and (3) advanced applications using these foundations, e.g., end-to-end analyses.

\section{Engineering Knowledge Base Methodology}

The top part of Figure 1 illustrates the use case scenario with the EKB framework for engineering a production automation system. In this example, there are two types of engineers (electrical engineer, software engineer) who come from to different engineering domains respectively. These roles use specialized engineering tools for their tasks. These tools contain local data sources, which produce and/or consume data with heterogeneous data structures. The EKB is used to facilitate the efficient data exchange between these engineering tools and data sources by providing a so-called "virtual common data model". Based on this data exchange, more complex tasks like model checking across tools are supported.

\subsection{Generic Engineering Knowledge Base Architecture}

This section describes the internal architecture of the EKB, as shown in the bottom part of Figure 1. The general mechanism of the EKB framework uses common engineering concepts identified beforehand as basis for mappings between proprietary tool-specific engineering knowledge and more generic domain-specific engineering knowledge to support transformation between these engineering tools [11]. In the following, the internal architecture of the $\mathrm{EKB}$ is described; the numbers directly refer to the numbered tags in Figure 1.

Extraction of Tool Data (1). As first step, the data elements contained in a particular tool need to be extracted in order to be available to the EKB framework. Since by now only a few engineering tools provide APIs for directly accessing the contained data, the export functionality of the tools is used. The exported data then is parsed and transformed into an internal format consisting of key-value pairs for each data attribute, which is easier to handle in the later steps.

Storage of Extracted Tool Data (2). The extracted and transformed key-value pairs are stored using a Java Content Repository (JCR) implementation, the so-called Engineering Data Base (EDB). For data storage, a tree structure is used, and additional functionality like versioning or roll-back is provided. The EDB is indexed and can be queried using Apache Lucene.

Description of Tool Knowledge (3a). The tool ontologies define the engineering-tool-specific, proprietary view on the information exchanged (e.g., a list of signals) in an integration scenario. This includes the view on the format of the information, but can also describe the meaning or the use of the specific view on the existing information, since there can exist multiple views for the same information. The most important part of this description is the definition of the exchanged information, i.e., the definition of the data structures either provided or consumed by a tool.

Description of Domain Knowledge (3b). The domain ontology contains the relevant shared knowledge between stakeholders in the particular application domain (in our case the industrial automation engineering domain) and hence represents the collaborative view on the information exchanged in an integration scenario. In addition, the domain ontology is the place to model standardized domain-specific information (e.g., the description of concepts used throughout an application scenario). The proprietary information of the engineering tools, which is defined in the tool ontologies, is mapped to the more general information of the domain ontology in order to allow the interoperability with other engineering tools. In contrast to a common data schema, the knowledge stored in the domain ontology is defined on a more general domain level compared to the knowledge stored in the tool ontologies. This particular domain-specific knowledge described in the domain ontology can easily be updated or transferred to other EKB-based integration scenarios residing in the same domain. This approach allows a broad spectrum of new applications in a particular domain to benefit from the described domain knowledge.

Mapping of Tool Knowledge to Domain Knowledge (4). Each data structure segment described in the tool ontology is mapped to either exactly one particular corresponding domain concept or domain concept attribute described in the domain ontology, or to e.g., all inherited sub-concepts of a target concept. In addition, the granularity of the mapped elements does not need to be the same, so that e.g., a concept can be mapped to the attribute of another concept, or vice versa. This defines the semantic context of the information contained in the segment and allows the detection of semantically similar information consumed and produced by other 


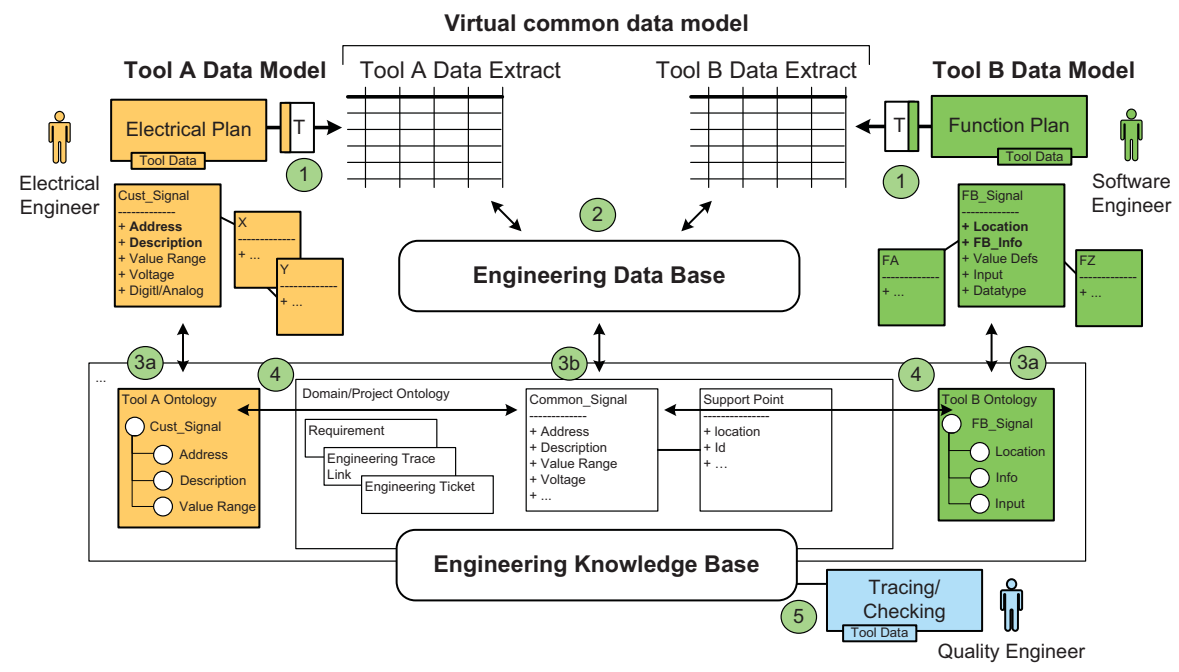

Figure 1. Overview Semantic Integration Approach [11].

engineering tools. In addition, the format of the information is described, enabling an automated transformation from source to target format.

Usage of the EKB (5). The mapping of concepts described in the tool ontologies to common concepts described in the domain ontology allows the creation of transformation instructions. These transformation instructions are the foundation to transform data structures between two engineering tools, because the engineering tools may label or format their data structures in different ways.

Due to the mappings between tool ontologies and domain ontology data structures that are semantically equal can be identified, because they are either aligned to the same domain concept or belong to the same tree segment in the concept tree described in the domain ontology. The transformation instructions can be defined in XML syntax and consist of at least one input and output data structure segment. The segments contain a unique ID and instructions, how the input segment is transformed to an output segment. There is a set of basic transformations that can be combined to more complex transformations, like changing the name of a segment, converting the format using converters, merging or splitting a set of input segments or querying external services for transformation [10]. Based on these transformations, more complex applications can be implemented which use the integrated data of the virtual common data model to perform advanced tasks like tracing of artifacts, consistency checking across tool boundaries, change impact analyses or notification of stakeholders in case of changes.

Now that we have described the general mechanism and the internal architecture of the EKB framework, the next step is the setup and configuration of the EKB framework. As described in [4], we suggest to use an enterprise service bus-based approach to integrate engineering tools by describing the data structures they produce and consume as services. The EKB framework acts as a component in the proposed technical integration solution, which performs its transformation service on the message transmitted using the enterprise service bus.

\section{Evaluation}

This section describes the prototypic realization of three typical EKB usage scenarios, namely data exchange between tools, model consistency checking across tool boundaries, and end-to-end analysis, in detail.

\subsection{Data Exchange Between Tools}

To cooperate the engineers have to exchange relevant parts of the data structures (i.e., information required in another tool should become available as soon as it has been saved in the original tool) in their tools with each other with the goal of a consistent overall view on certain aspects in the project, e.g., when producing a specification for a subcontractor. Currently, every role uses organization-, domain-, and tool-specific data formats and terms, thus the data exchange takes considerable expert knowledge on the receiving end to make sense of the incoming data, typically as large PDF document or tool-specific import file [11].

Common Repository Approach. The exchange of data structures originating from different engineering tools using a common repository requires a set of prerequisites. Either, all participating tools need to agree on a common data schema used for the data structure exchange. All exchanged information is then structured according to this schema. While this is even hard for tools originating from the same engineering domain, it becomes nearly impossible for tools originating from different and typically heterogeneous engineering domains. In addition, changes to one or more of the engineering tools regularly require an update of the common schema, which then needs to be forwarded to the other engineering tools which use this schema. So the major functionality of the common repository is to store all information, while at the same time providing point-to-point integration between all participating tools using converters for each possible combination of the tools. Once set up and configured properly, this data exchange method has a low delay, i.e., information made available by an engineering tool is available for all other engineering tools that need these information. However, the configuration of this approach requires high effort, since converters need to be written for all needed pairs of $\mathrm{n}$ engineering tools, with $\mathrm{O}\left(\mathrm{n}^{2}\right)$ required converters. In addition, the common repository is inflexible and fragile in case of changes of single engineering tools, since converters need to be adapted or complete rewritten in this case [11].

Engineering Knowledge Base (EKB) Approach. A first step in using the EKB framework is the identification of common concepts used in the participating engineering tools. As a next step, the proprietary tool-specific knowledge is mapped to the more general common concepts. Based on these mappings, the EKB framework semi-automatically generates transformation instructions for transforming data structures between tool-specific formats. This semi- 
automated generation exploits the mappings stored in the EKB and makes suggestions for possible transformations to be reviewed by a human expert. The human expert then can revise the suggested transformation, change them or add new or more complex transformation rules manually. Since for each of the $n$ participating engineering tools a single transformation instruction is required, the number of overall needed transformation instructions is $\mathrm{O}(\mathrm{n})$ [11].

While the EKB framework requires similar or at most slightly higher effort for setup and configuration compared to the common repository approach, new benefits come from using ontologies for storing the engineering knowledge. The ontologies enable the semiautomated generation of the required converters, both initially and when engineering tools evolve. The number of required converters is also smaller with $\mathrm{O}(\mathrm{n})$ converters for $\mathrm{n}$ engineering tools. Further, once set up, the delay of the data exchange method is similar to the delay using the traditional common repository based approach [11].

\subsection{Model Consistency Checking Across Tool Boundaries}

Model checking, i.e., the validation of model data elements regarding their integrity and consistency, typically is performed at project milestones before the model elements can be used in the next stage of engineering. For a safety-critical domain such as the production automation domain, model checking is required for obtaining relevant system certifications. Currently, model checking is limited to single engineering tools or engineering domains. In addition to syntactical checks, plausibility checks of model elements regarding their usage in other engineering domains are needed.

An example for consistency and integrity checks of model changes is a hardware pump which supports a certain number of input/output signals (I/Os), and which is controlled by a pump control software using either analog or digital signal processors. Analog signal processors can handle $8 \mathrm{I} / \mathrm{Os}$, while digital signal processors can handle $64 \mathrm{I} / \mathrm{Os}$. If the signal processor type is changed in the pump control software model, it needs to be validated whether the new signal processor type can handle all available I/Os of the hardware pump. Respectively, if the I/Os are changed (e.g., new I/Os added) it has to be checked whether they all can be controlled using the chosen signal processor type of the pump control software. Another example for the derivation of runtime functionality for automated testing and monitoring is again a hardware pump which can handle approximately 1000 liters per hour. A time-based analysis of the events originating from the reservoir located behind the hardware pump could show impossible conditions or sensor states, e.g., if the reservoir capacity of 10000 liters is reached within 5 hours starting from an empty condition [11].

Common Repository Approach. Using a common repository enables to perform advanced checks regarding the data structures of more than one engineering tool, such as checking the consistency of single data structure elements across tool boundaries or analyzing the possible impact of changes to data structures belonging to a specific engineering tool on the data structures of other engineering tools. The major drawback of this approach of performing model checks is the need for manual involvement of human experts. The experts need to explicitly define the checks and select the data they want to include in these checks. This definition needs to be updated after every change to the involved data elements. Additionally, the nature of the common repository allows only for syntactical checks (e.g., the availability of all obligatory data fields or the validity of data types regarding a certain data schema) of the data, but not for other checks such as regarding the semantic correctness or plausibility of data structures. Other checks, such as checks regarding logical connections of data elements, are not supported out of the box using a common repository, since the data elements in the repository are stored unaltered and without meta-information.

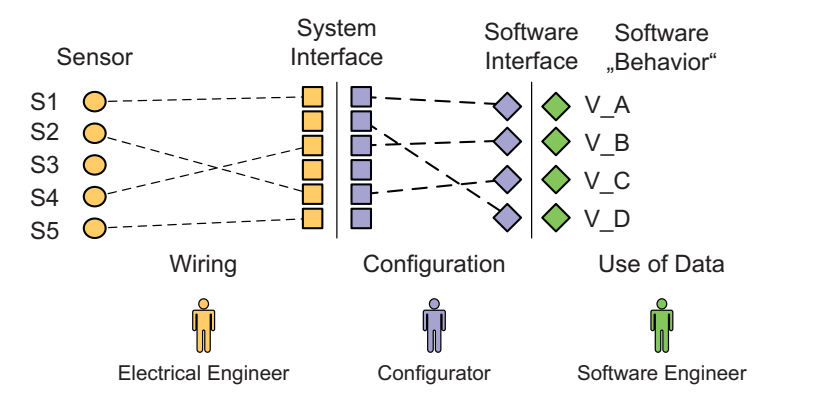

Figure 2. Overview End-to-End Analysis [12].

However external analysis tools can use the data elements stored in the common repository for performing such model checks [11].

EKB Approach. The EKB framework enables automated checks regarding both syntactical issues as well as plausibility checks regarding semantic correctness of data structures. The EKB framework exploits the querying capabilities of ontologies to allow even more advanced checks, such as checks regarding completeness of available information. Human experts define checks regarding specific domain or tool-specific concepts, which are then onthe-fly transformed into checks regarding tool-specific data structures accordingly. The results are then collected and again transformed into the domain concept level, allowing experts both to define checks as well as to view the results of the defined checks in their well-known syntax, terminologies and notations [11].

\subsection{End-to-End Analysis}

In distributed engineering in heterogeneous environments, typically a set of different models is used along the engineering chain. In order to ensure validity and consistency of the overall engineering process, it is important to ensure that required data fields can be enforced during the whole lifecycle of the engineering chain [12].

In an automation systems engineering context, this may be defined as a list of hardware sensors and software variables (as shown in Figure 2), which are connected to a system interface by virtual links in models or by wiring in the real-world. Internally, the signals are mapped from the system interface to a software interface, where these signals are represented as variables. A typical consistency and validity check may be used to check whether there exist any incomplete chains between variables and sensors.

If we assume that the data originating from the three different engineering disciplines is not homogeneous, but rather is only available in heterogeneous form, simple SQL-based approaches presented do not work anymore. A solution approach would be to provide mapping tables in the database, which store the mappings between the attributes defining the links between entities. However, this results in both an increasing complexity of the SQL statements, as well as in the need to adapt these mapping tables each time attribute values are changed.

We now model this scenario using the EKB framework. There are three different layers, namely the domain ontology, the tool ontologies and the instance data layer. In the domain ontology, the general attributes (the so-called overlapping engineering concepts) and the relations of the concepts electric, configuration and software are modeled. In the tool ontologies, all attributes of the toolspecific concepts electric, configuration, and software are modeled. In addition, the mappings between the tool ontologies and the attributes of the generic concepts modeled in the domain ontology are described. Using this model allows us to define queries on the domain ontology layer. 


\section{Conclusion}

Software-intensive systems in business IT and industrial automation and software engineering projects bring together experts from several engineering domains and organizations, who work in a heterogeneous engineering environment with a wide range of models, processes, and tools that were originally not designed to cooperate seamlessly [14]. A core question is how to integrate data models across tools and domain boundaries. Current semantic engineering environment integration is often ad hoc and fragile, making the evolution of tools and re-use of integration solutions across projects risky [7][13].

In order to reach the common goal of developing software products in the engineering team, it is important to share the necessary knowledge for common work processes between engineering domain experts [14]. However, this knowledge is often only implicitly available and therefore inefficient to share, resulting in time-consuming repetitive tasks; often it is hard or even impossible to create and maintain common shared knowledge repositories. A method and platform for making expert knowledge explicit and efficiently shareable is needed in order to support quality and project managers in their data analyses based on engineering knowledge and concrete data in the engineering tool models, which is currently achieved using inefficient or fragile approaches.

In this paper, we described the Engineering Knowledge Base (EKB) methodology for supporting engineering environment integration in multi-disciplinary engineering projects with a focus on providing links between data structures of engineering tools and systems to support the exchange of information between these tools and thus making software and systems engineering more efficient and flexible. Since standards are hard to apply in projects where experts from different organizations participate, who have invested into different kinds of local standards or approaches, these experts may use their well known local tools and data model, and additionally can access data from other tools in their local syntax. The EKB is located on top of a common repository and stores explicit engineering knowledge to support access and management of engineering models across tools and disciplines by providing a) data integration by exploiting mappings between local and common engineering concepts; $b$ ) transformations between local engineering concepts by following these mappings; and c) advanced applications using these foundations, e.g., end-to-end analyses. As a result experts from different organizations may use their well known local tools and data model, and additionally can access data from other tools in their local syntax. Since the engineering project participants by now already work together, they already use common knowledge for their project tasks. By using the EKB framework we make this existing knowledge explicit and machineunderstandable, and therefore can automate on project level tasks that build on this explicit and machine-understandable knowledge. Furthermore, using the EKB framework allows a more generic definition and execution of model checks on an application domain level, and additionally enables more advanced checks regarding the plausibility and semantic correctness of data structures by exploiting the querying capabilities of ontologies.

Further Work. After evaluating the feasibility of the EKB approach next steps are studies that extend the scale of the data models involved and address the following research issues: efficient import of data models into EKB ontologies, robust semantic mapping, and performance of the EKB ontologies for larger data sets. Practical issues such as effort and defect rates for setting up an EKB with larger-scale data models and for semantic mapping with engineering experts need to be explored in setting with industrial experts. Once the EKB framework is set up properly, additional use cases can be supported in order to make software and systems engineering more efficient. Planned use cases to evaluate are the tracing of requirements between data structures across tools as well as the support for change management across tools.

\section{Acknowledgments}

This work has been supported by the Christian Doppler Forschungsgesellschaft and the BMWFJ, Austria. This work has been partially funded by the Vienna University of Technology, in the Complex Systems Design and Engineering Lab.

\section{References}

[1] D. Assmann, J. Drr, M. Eisenbarth, M. Hefke, M. Soto, P. Szulman, and A. Trifu. Using ontology-based reference models in digital production engineering integration. In 16th IFAC World Congress, Prague, Czech Republic, 2005.

[2] S. Bergamaschi, S. Castano, and M. Vincini. Semantic integration of semistructured and structured data sources. SIGMOD Rec., 28(1):5459, 1999.

[3] P. A. Bernstein and U. Dayal. An overview of repository technology. In 20th International Conference on Very Large Data Bases, pages 705-713. Morgan Kaufmann Publishers Inc., 1994.

[4] S. Biffl, A. Schatten, and A. Zoitl. Integration of heterogeneous engineering environments for the automation systems lifecycle. In IEEE Industrial Informatics (IndIn) Conf., pages 576-581, 2009.

[5] A. Doan and A. Halevy. Semantic integration research in the database community: A brief survey. AI Magazine, 26(1):83-94, 2005.

[6] C. H. Goh. Representing and Reasoning about Semantic Conflicts in Heterogeneous Information Systems. PhD thesis, MIT, 1996.

[7] A. Halevy. Why your data won't mix. Queue, 3(8):50-58, 2005.

[8] G. Hohpe and B. Woolf. Enterprise Integration Patterns: Designing, Building, and Deploying Messaging Solutions. Addison-Wesley Professional, 2004.

[9] P. Kruchten. The rational unified process: an introduction. AddisonWesley Longman Publishing Co., Inc. Boston, MA, USA, 2000.

[10] T. Moser, K. Schimper, R. Mordinyi, and A. Anjomshoaa. Samoa a semi-automated ontology alignment method for systems integration in safety-critical environments. In 2nd IEEE International Workshop on Ontology Alignment and Visualization (OnAV'09), pages 724-729, Fukuoka, Japan, 2009.

[11] T. Moser, S. Biffl, W. D. Sunindyo, and D. Winkler. Integrating production automation expert knowledge across engineering stakeholder domains. In International Conference on Complex, Intelligent and Software Intensive Systems (CISIS 2010), pages 352-359, Krakow, Poland, 2010. IEEE.

[12] T. Moser, D. Winkler, and S. Biffl. Engineering analyses across domain boundaries using the engineering knowledge base framework. Technical Report (available online at: http://www.ifs.tuwien.ac.at/files/Engineering Analyses across Domain Boundaries using the Engineering Knowledge Base Framework Technical Report.pdf), 2010.

[13] N. F. Noy, A. H. Doan, and A. Y. Halevy. Semantic integration. AI Magazine, 26(1):7-10, 2005.

[14] W. Schfer and H. Wehrheim. The challenges of building advanced mechatronic systems. In 2007 Future of Software Engineering - International Conference on Software Engineering, pages 72-84, Washington, DC, 2007. IEEE Computer Society.

[15] D. Trowbridge, U. Roxburgh, G. Hohpe, D. Manolescu, and E. Nadhan. Integration Patterns. Patterns \& Practices. Microsoft Press, 2004.

[16] H. Wache, T. Vgele, U. Visser, H. Stuckenschmidt, G. Schuster, H. Neumann, and S. Hbner. Ontology-based integration of information-a survey of existing approaches. In Workshop on Ontologies and Information Sharing (IJCAI-01), pages 108-117, Seattle, USA, 2001.

[17] T. Weilkiens. Systems engineering with SysML/UML: modeling, analysis, design. Morgan Kaufmann, 2008. 